\title{
Methodology for testing a system for remote monitoring and control on auxiliary machines in electric vehicles
}

\author{
Vasil Dimitrov ${ }^{1, *}$, and Petko Kostadinov ${ }^{1}$ \\ ${ }^{1}$ Todor Kableshkov University of Transport - Sofia, Department of Power Engineering and Electrical Equipment in Transport, \\ Faculty of Telecommunications and Electrical Equipment in Transport, 158 Geo Milev St., 1574 Sofia, Bulgaria
}

\begin{abstract}
A laboratory system for remote monitoring and control of an asynchronous motor controlled by a soft starter and contemporary measuring and control devices has been developed and built. This laboratory system is used for research and in teaching. A study of the principles of operation, setting up and examination of intelligent energy meters, soft starters and PLC has been made as knowledge of the relevant software products is necessary. This is of great importance because systems for remote monitoring and control of energy consumption, efficiency and proper operation of the controlled objects are very often used in different spheres of industry, in building automation, transport, electricity distribution network, etc. Their implementation in electric vehicles for remote monitoring and control on auxiliary machines is also possible and very useful. In this paper, a methodology of tests is developed and some experiments are presented. Thus, an experimental verification of the developed methodology is made.
\end{abstract}

\section{Introduction}

Speed regulation of auxiliary machines in electric vehicles (pumps, fans, air coolers, compressors, etc.) is often unnecessary. These drives need to be started and stopped smoothly but their speed can be constant through the operating modes. Asynchronous motors controlled by soft starters can be used in those cases - their implementation enhances the starting performance of the drives by allowing the motor to start gradually and smoothly in a controlled manner [1-6]. Contemporary soft starters can be set up to meet the requirements of the individual application and the motor is adjusted to the machine's load behaviour $[4,5]$.

Therefore, the study and examination of soft starters is very important. A laboratory system has been designed and built and remote monitoring and control by an intelligent energy meter and PLC has been provided [7]. In this paper, a methodology of tests has been developed and verified.

\section{Possibilities of testing and research}

The existence of PLC with touch panel and intelligent energy meter Sentron PAC3200 connected to a PC in the developed laboratory system allows for simultaneous drive control, monitoring and parameters' tracing and capturing through the relevant software product Powerconfig V3.7 installed on the PC [7-9]. Thus this system offers various possibilities of tests and research, for example:

- Training the programming skills for setting-up the soft starters, intelligent measuring devices, PLCs and touch screens, as well as communication between these devices and the $\mathrm{PC}$ - knowledge of the relevant software products is necessary;

- Remote monitoring of the drive operation and analysis of the electrical system's current consumption and power flows;

- Capturing the dynamic characteristics when starting and stopping the drive at different settings and loads;

- Determination of the correlation between the soft starter settings and the drive performance and energy parameters (current, power, voltage, power factor, efficiency, THD, etc.);

- Study of the impact of start-up and ramp-down time on drive performance;

- Synthesis of algorithms for optimal control of the drive systems;

- Evaluation of the PLC's possibilities of using it in the drive systems for efficiency optimization;

- Examination of the asynchronous drive at variable load, etc.

\subsection{Remote monitoring and analysis of the drive's parameters}

The Sentron PAC3200 device must be connected to the $\mathrm{PC}$ and the Powerconfig V3.7 software must be run $[8,9]$. This software package is a combined service and commissioning tool for Sentron measuring devices. It offers the possibility for remote parameterization of the device, as well as monitoring and documentation of the system parameters. The measured values can be observed in real time in numerical and graphic form - the moment values are written and the graphics can be plotted (Fig. 1).

* Corresponding author: vdimitroff@abv.bg 


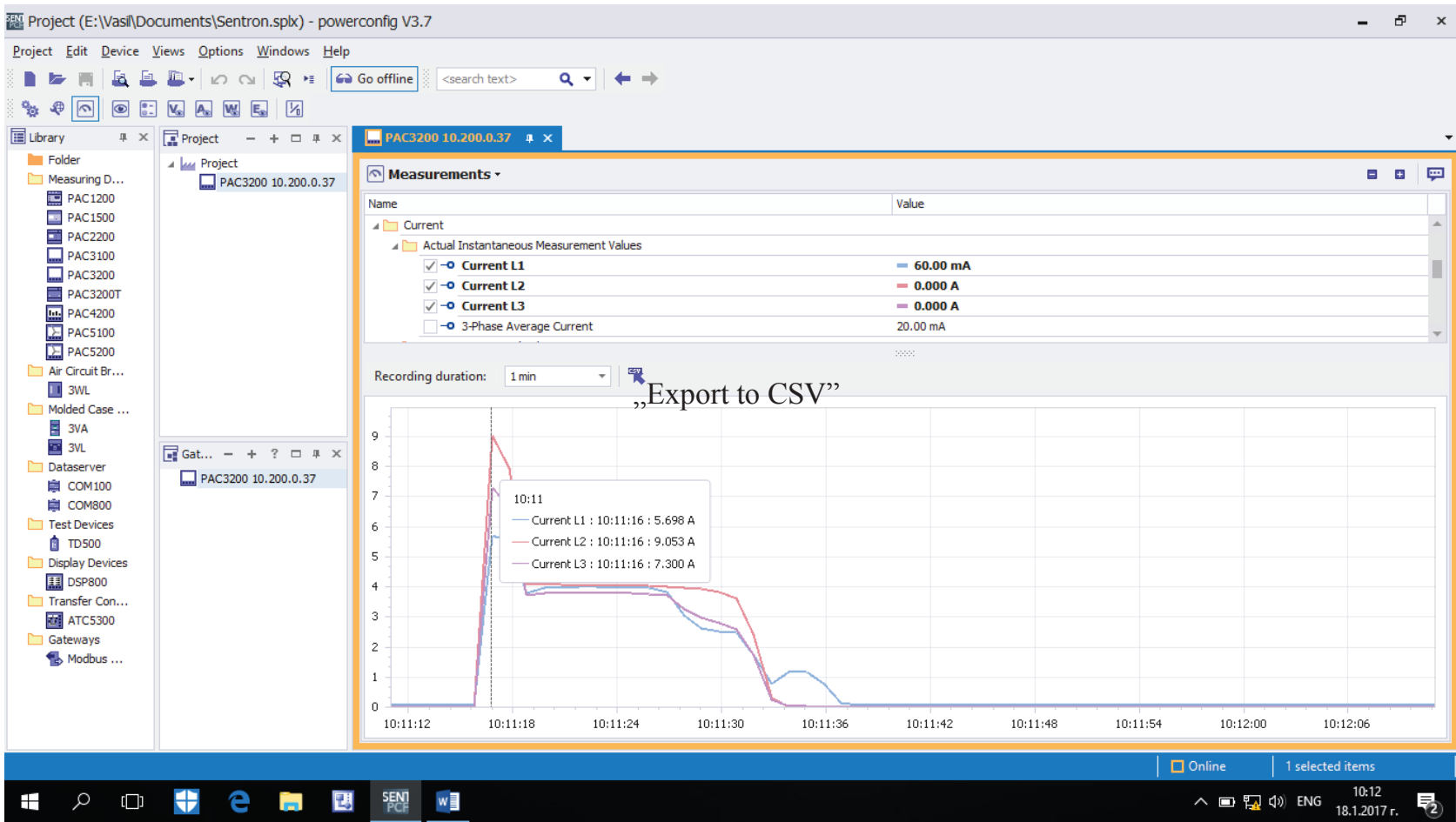

Fig. 1. Software Powerconfig V3.7 - current monitoring.

All the relevant system parameters can be shown on the PC display selected through the menu: voltage (phaseneutral and phase-phase), currents, active, reactive and apparent power (per phase and total), power factor (per phase and total), frequency, total harmonic distortion (THD) for voltage and current, phase unbalance (voltage), etc. The date, time and value of the selected parameters are displayed in real time. The refresh time could be changed (the minimum value is $330 \mathrm{~ms}$ ) and the differences could be observed. Read-out and saving of message lists, load profiles, and histories is also possible.

The variation of the motor currents during different regimes is shown in the Fig. 1. The big values of the starting currents are clearly visible. The currents are constant during the steady-state operation and decrease slowly after the Stop command. The difference between the currents is caused by the unbalanced three-phase voltage and the increased value $(\sim 1 \mathrm{~A})$ of the current I1 after a Stop command is generated by affecting the electrodynamic brake. Thus these graphics show a proper operation of the drive.

\subsection{Capturing the dynamic characteristics}

The Powerconfig V3.7 software provides the opportunity to export data to Excel tables using the "Export to CSV" button (see Fig. 1). The duration of recording has to be defined in advance. The data can be processed in Excel and many graphics can be plotted and analyzed.

The methodology of capturing the dynamic characteristics $I=f(t)$ when starting and stopping the drive are as follows:

1) The laboratory system must be switched on by the breaker. Three phase voltage is displayed on the Sentron
PAC3200. Many other parameters could be visualized by pressing F2 or F3 function keys [8]. These parameters can also be seen on the PC monitor.

2) Direction of the motor rotation has to be selected (by $F$ or $R$ buttons).

3) The acceleration time $t_{a c c}$ has to be set by the potentiometer. The deceleration time $t_{d c c}$ and the initial voltage $U_{\text {in }}$ can be also set by other potentiometers. The Boost-function could be also activated by the switch on the Touch Panel.

4) Sentron PAC3200 has to be set to display the currents. Min. / max. values stored in the memory can be checked using function key F1 and they have to be cleared by pressing key F3.

5) The drive starts by pressing the button on the Touch Panel. The values of the currents have to be observed. If there are voltmeters connected between the soft starter and drive, the values of the three phase motor voltage could also be observed. Acceleration time can be measured by a chronometer. The yellow indicator of the soft starter lights up after $10 \mathrm{~s}$ - the motor has received maximum power supply.

6) The maximum currents reached during starting can be displayed using function key F1. They may be compared to the values shown in the graphics.

7) The drive can be stopped by pressing the button on the Touch Panel. After the soft starter is switched off the drive could be stopped by means of the $B r$ button if it is necessary.

8) The time $t_{a c c}$ or the initial voltage $U_{i n}$ must be changed and tests 3) - 7) have to be performed again.

After the end of the recording the data are exported in an Excel table and the graphs can be plotted. The threephase current is shown in Fig. 2. 


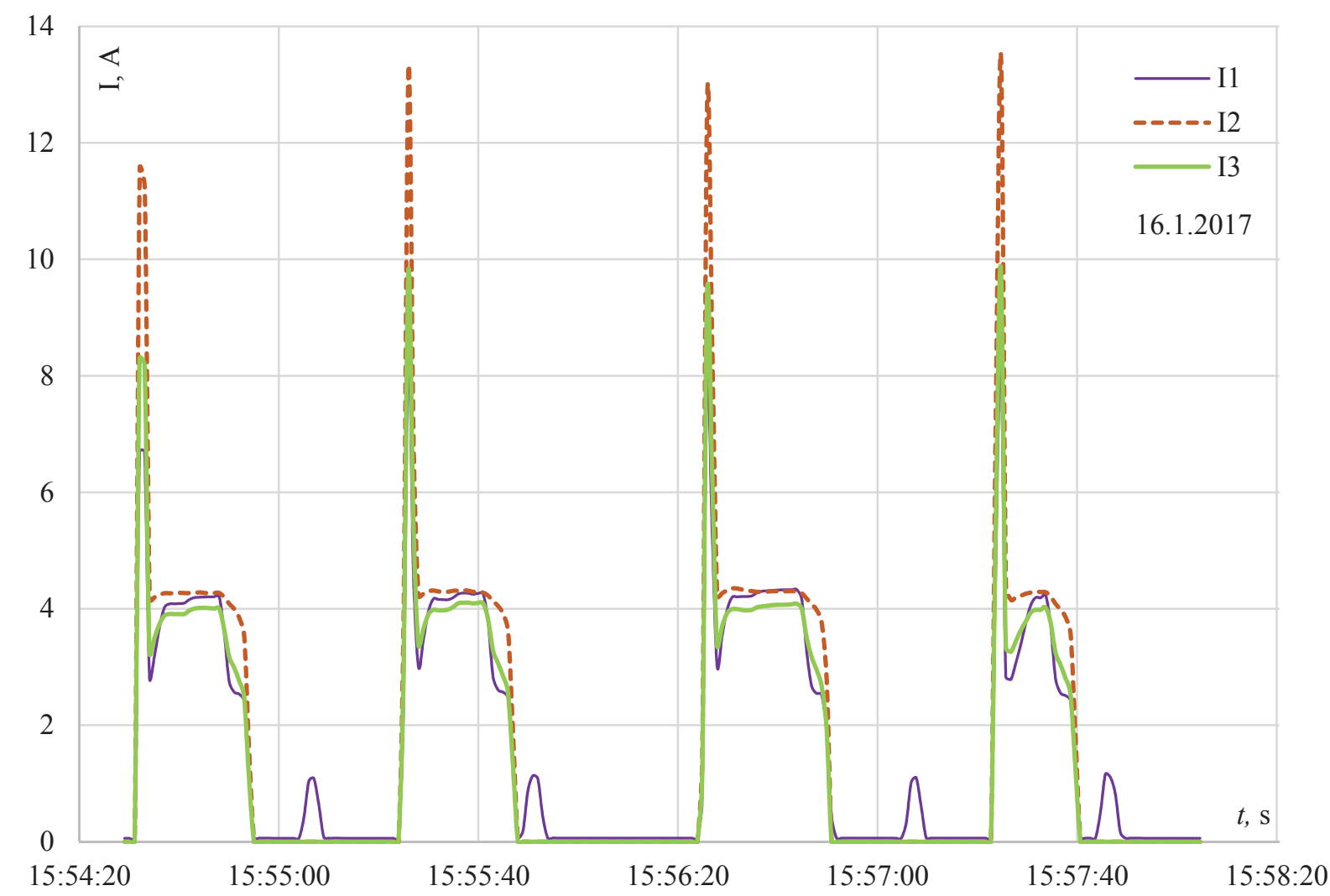

Fig. 2. Captured dynamic characteristics $I=f(t)$ at different settings of the acceleration time $t_{a c c}$ and initial voltage $U_{i n}$.

All the relevant system parameters changes (P, Q, $\cos \varphi$, THD, etc.) can be captured by similar methods.

It is clearly visible in Fig. 2 that the big values of the starting currents are different with changes in the acceleration time $t_{a c c}$ and initial voltage $U_{i n}$. The currents are independent of the soft starter settings during the steady-state operation and decrease slowly after the Stop command.

\subsection{Determination of the correlation between the soft starter settings and the drive performance}

The developed methodology of tests allows to determine the correlation between the parameters of the drive (starting current $I_{s t}$, power factor $\cos \varphi$, efficiency $\eta$, THDI, THD-U, power - active $P$, reactive $Q$, apparent $S$, etc.) and the settings of the soft starter:

$$
\begin{aligned}
& I_{s t}=f\left(t_{a c c} ; t_{d c c} ; U_{i n}\right), P=f\left(t_{a c c} ; t_{d c c} ; U_{i n}\right), \\
& Q=f\left(t_{a c c} ; t_{d c c} ; U_{i n}\right), S=f\left(t_{a c c} ; t_{d c c} ; U_{i n}\right), \\
& \cos \varphi=f\left(t_{a c c} ; t_{d c c} ; U_{i n}\right), \eta=f\left(t_{a c c} ; t_{d c c} ; U_{i n}\right), \\
& T H D_{I}=f\left(t_{a c c} ; t_{d c c} ; U_{i n}\right), T H D_{U}=f\left(t_{a c c} ; t_{d c c} ; U_{i n}\right) .
\end{aligned}
$$

The data obtained during the appropriate tests must be previously exported to an Excel table and the graphs have to be plotted. In this paper, the correlation between the acceleration time and starting current at different settings of the initial voltage is determined. Data and graphs analogue to these shown in the Fig. 2 have been used. The methodology of determining this correlation is as follows:

1) The average values of the starting current at different settings must be calculated:

$$
I_{s t}=\frac{I_{1 s t}+I_{2 s t}+I_{3 s t}}{3} .
$$

2) The graph representing the correlation between the starting current and acceleration time at constant initial voltage has to be plotted.

3) A family of characteristics can be obtained at various settings of the initial voltage as a percentage of the nominal voltage (Fig. 3 ).

It is obvious that the change of the acceleration time setting, respectively the rate of the supply voltage increasing, results in a different maximum reached value of the starting current. When acceleration time is increased, the starting current is smaller, and when the initial value of the supply voltage is increased, the starting current also increases.

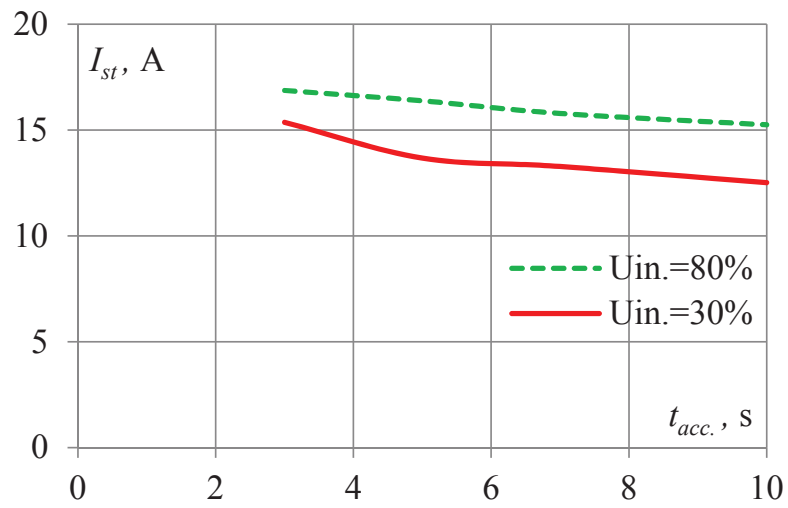

Fig. 3. Impact of the acceleration time $t_{a c c}$ on the starting current $I_{s t}$ at different settings of the initial voltage $U_{i n}$. 


\subsection{Study of the impact of start-up time on the drive performance}

Using the described methodology, the correlations between the acceleration time and the power factor $P F$ (Fig. 4) and the coefficient THD-I (Fig. 5) have been plotted on graphs. It can be noted that the load of the drive is constant during the tests because the load of the auxiliary machines in electric vehicles does not change during their operation.

It can be seen that the power factor improves when reducing the acceleration time. This interdependence can be explained by the bigger starting torque developed by the asynchronous motor for a short time, and hence the greater value of the active power.

The THD-I value deteriorates as the acceleration time increases. This interdependence can be explained by the operation of the thyristor voltage regulator. Longer acceleration time is provided by increasing the unlocking angle $\alpha$ of the thyristors, which leads to a distortion of the sinusoidal form of the current $[1,2]$.

Therefore, changing the settings has a different impact on the drive parameters. The optimal solution for each particular case could be achieved but a complex research and testing have to be done.

Similar tests can be conducted to determine the effect of the Boost function and the deceleration time on the drive performance and energy efficiency. For example, the energy consumption during transient regimes of the auxiliary machines could be optimized: the initial voltage

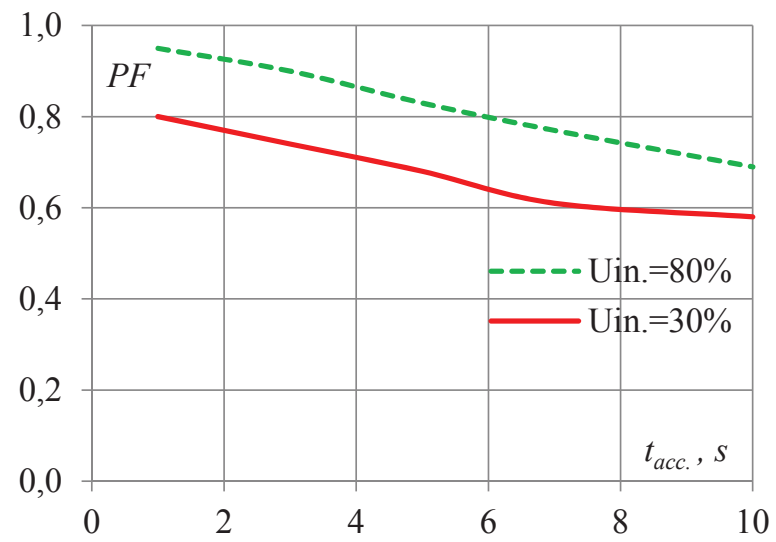

Fig. 4. Impact of the acceleration time $t_{a c c}$ on the power factor $P F$ at different settings of the initial voltage $U_{i n}$.

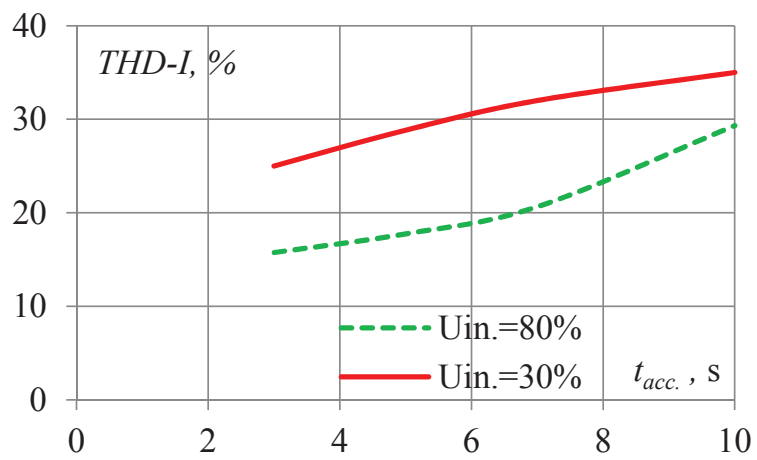

can be reduced when a centrifugal mechanism is driven (fan, pump, rotational compressor, etc.) because its starting torque is very low. However, if a piston compressor is driven, a big value of the initial voltage has to be chosen and even the boost function may be activated.

\section{Conclusion}

A laboratory system has been designed and built using contemporary devices. An asynchronous motor is controlled by a soft starter and opportunities for remote monitoring (by an intelligent energy meter) and control (by a PLC and Touch panel) have been provided.

This laboratory system offers various possibilities for research and implementation into practice. Methodologies for different tests are developed. The impact of soft starter settings on drive performance and energetic parameters amendment could be examined. The interdependencies obtained during the experiments are similar to the characteristics known in the technical literature [1-3]. Thus an experimental verification of the described methods has been carried out.

If such a system is involved in electric vehicles for remote monitoring and control on auxiliary machines, energy efficiency and reliability will increase. The starting current will be reduced and the power factor and THD could be optimized in comparison with the direct connection of the motor to the grid. The optimal setting of the soft starter for each particular case must be done in advance. As a result of real time monitoring of drive performance any failure or damage can be discovered immediately and the motor can be instantly switched off from a distance by the operator in the control center.

\section{References}

1. A. Hughes, Electric Motors and Drives Fundamentals, Types and Applications. Third edition, Elsevier Ltd (2006)

2. U. Bakshi, M. Bakshi, Electrical Drives and Control, Technical Publications Pune, India (2009)

3. I. Boldea, A. Nasar, Electrical Drives, CRC/Taylor \& Francis Group, NY (2006)

4. The essential guide of Automation \& Control (Schneider Electric, 2012)

5. Soft Starts for Single-Phase and Three-Phase Asynchronous Motors, Catalog, Schneider El (2011)

6. E. Dimitrova, Building automation and control systems, "MTC", 14 3/2, art. ID: 1395 (2016)

7. V. Dimitrov, Design and setting up of a system for remote monitoring and control on auxiliary machines in electric vehicles, BulTrans Proceedings (2017)

8. SENTRON PAC3200, Technical specification, Siemens (2011)

9. http://w3.siemens.com/powerdistribution/global/en/lv /portfolio/pages/powerconfig.aspx

Fig. 5. Impact of the acceleration time tacc on the coefficient THD-I at different settings of the initial voltage $U_{i n}$. 